\title{
Research And Implementation Of Network Monitoring Service Model Based On SOA Architecture
}

\author{
Xiao-YanLiu ${ }^{1, a}$,Qing-RuSui ${ }^{2, b}$ \\ ${ }^{1}$ Changchun University Of Science AndTechnology, Changchun130600, China. \\ ${ }^{2}$ Changchun University Of Science AndTechnology, Changchun130600, China. \\ a932684698@qq.com, ${ }^{\mathrm{b}} 404409709 @ q q . c o m$
}

Key words: SOAarchitecture, Network management, Service, The service bus.

\begin{abstract}
Network security management problems highlighted increasingly. This paper proposes a model of network monitoring service based on service-oriented architecture (SOA), Because of its low coupling,has nothing to do with the platform , SOA is good to solve the problem of the system flexibility and interoperability.Apply SOA to network monitoring management, to further improve the efficiency of network management.
\end{abstract}

\section{Introduction}

Network security is an important task of the network age,to solve the problem of network security network monitoring software and system arises at the historic moment. Wecan say that the network monitoring service system to a certain extent in the corresponding field does play a role. But with the rapid growth of network traffic and further development of information,management software and network security system also exposed some shortcomings and defects[1].For example the independent network management software,poor reusability and maintenance is not convenient.Leading to an increase in the cost of network management, the serious influence and reduce the efficiency of network management.

Service-oriented Architecture SOA is a software design framework, which is different from the traditional software design framework. It will be the application of different functional units (called services) through well-defined interfaces and contracts between these services. This makes the building in a variety of services in such a system can be in the form of a unified and general interaction.SOA technology application in the field of network management,give full play to the high scalability and adaptability of SOA,old software can share information to work together, the newly developed heterogeneous software can quickly integration. Separating the business logic and the specific implementation technology will make more conducive to upgrading of software system to adapt to the rapid development.

\section{Traditional Network Monitoring Model Analysis}

Network monitoring is one of the most basic parts of the network management. The purpose of network monitoring is to collect information on network elements state and behavior.Collect information can be divided into three types: static, dynamic and statistical information. Static information refers to the general does not change with time and configuration information;Dynamic information is related to the network time, information changes over time;Statistics are based on dynamic information according to certain rules and produce information.Network monitoring tools, as a very important part of network management platform, is mainly responsible for collecting the network state and each equipmentbehavior information .Traditional network monitoring tools are based on SNMP model,can do some simple network state information retrieval.but not for information sharing between the monitoring module.

In order to analyze the characteristics of the traditional network monitoring model based on the SNMP. We have a FTP, WEB and DNS sub network system as an example. There are 3 kinds of 
server in the network, which provide FTP service, Web service and DNS service. The realization of these services dependent network server system and their operation at the same time. Network management platform need to run 5 monitoring module to monitor the network, in order to achieve effective management of the whole network.The five monitor modulesare a separate operation, the collected data is stored by the storage module, analyzing by the analysis module. The monitoring model with traditional centralized data collection and storage; monitoring module is independent of each other, not aware of each other etc. With the increase of the monitored system, the complexity will increase as the monitoring platform. In the actual network management, management system using the traditional monitoring model is often not very good complete management function.

With the development of the Internet, complex of network structure, large amount of information,the network monitoring is becomemore difficult. The traditional based on SNMP network monitoring tool not timely, accurately reflect the network running status, less and less able to meet the demand,So to discuss a new model of network monitoring.

\section{Service Oriented Architecture (SOA)}

The Definition Of SOA.SOA is a service-oriented architecture, is a component model, it is a kindof popular software design architecture. It will be the application of different cells through these well-defined interfaces and contracts between them, these units are called services.Differernt services through a simple and precise definition of the interface for communication[3]. SOA architecture contains the service provider, service registry, and service users and three kinds of action about the service:publish, find, and bind. Among them, the service provider to release and response service,service registry search service provider, Service users to find and use services provided by the service provider.

The Characteristics Of SOA.SOA is a loose coupling and coarse granularity, the architecture of the communication between the service does not involve the underlying programming interface and the communication model[4]. SOA is an architecture, it focuses on the software function, that is, a how is the function of the software need to be provided by another software, how does the complex function can be made from a mix of simple function, according to actual needs can integrate multiple software system into a new software. Service is can be updated according to the needs of specific organizations become larger and the service of a complete business unit, it can be made up by one or more components.

\section{Technology AndRealization Of The Network Monitoring Service Model Based On SOA}

The KeyTechnology Of SOA. Currently implementing an SOA approach is generally recommended Web services, basic workflow is shown in figure. Web Services is a collection covers interoperability standards [5].These standards defines for the communication protocol, and defines the interface used to illustrate the service and the service contract format. As a result of the numerous well-known enterprise research and development, now has more than dozens of Web Services standard, and have different standards organization in this paper, the necessary information. These Web Services standards covered almost distributed computing and remote procedure/function/service call all areas. Here are five basic Web Services standards.

- XML(extensible Markup Language) using the tags to define the content, is used to describe the model, the format and data types of common format. It is the foundation of other Web Services standards.

- HTTP (HTTPS) is the basic protocol of Internet use. HTTP (HTTPS) is to use the Internet technology, through the network to send Web Services may be one of the protocols.

- WSDL (Web services Description Language) is used to define the Service interface. WSDL is used to describe a web service or terminal of an XML language. It can describe two different aspects of service: the signature of the service (name and parameters), as well as the service binding and deployment details (protocol and location). 
- SOAP (simple object access protocol is simple object access protocol) is a protocol based on XML. Web Service using SOAP protocol implementation across different programming languages and operating system platform, it through the HTTP protocol to send request and receive results, using XML format encapsulation, and increases the specific HTTP header, to illustrate the content of the HTTP message format. These particular HTTP headers and content of the XML format is SOAP protocol.

- UDDI (Universal Description Discovery and Integration) is the management of Web Services standards, the implementation of the service to find the registration and other Services.

Construct Network Monitoring Service Model Based On SOA.The network monitoring service model based on SOA implementation process as shown in Figure 1.

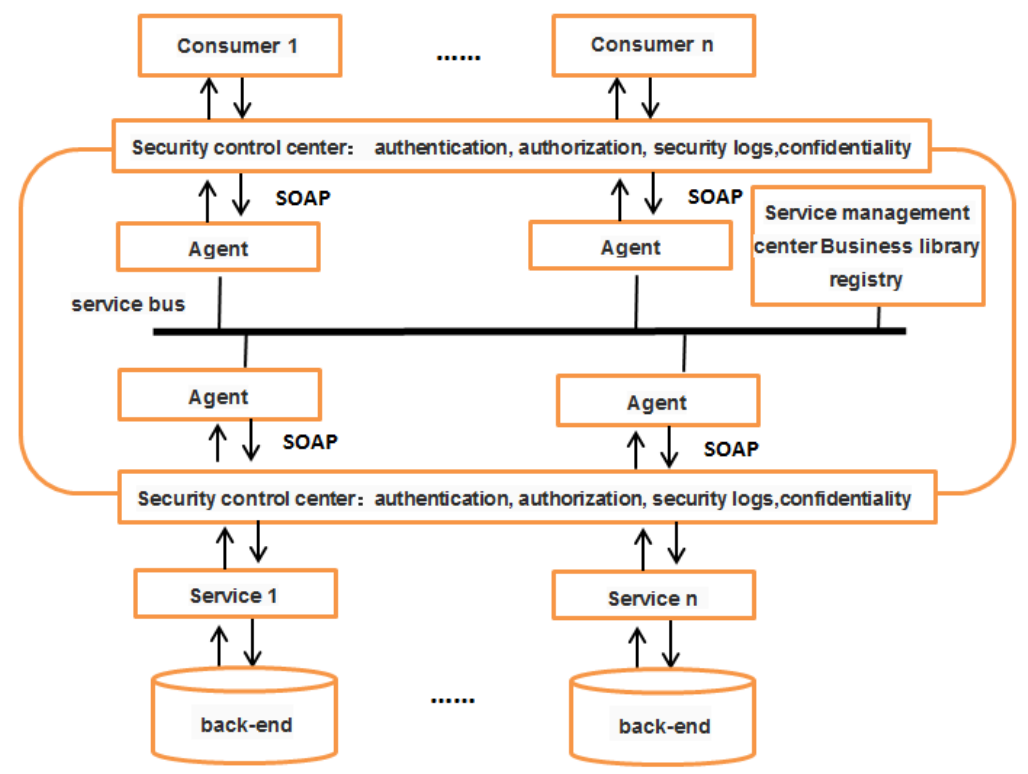

Figure1Model of network monitoring based onSOA

- $\quad$ Service bus : It is the infrastructure of this system, its main task is to provide connectivity, data format conversion, intelligent routing, treatment safety, reliability, service, management, testing and logging, etc.

- Security control center: in order to improve the safety performance of the system, model is introduced into safety control center. Different consumers have different permissions, the service bus through the security control center to identify the consumer at the same time control the permissions to invoke the service. The service bus through the security control center to identify the provider. At the same time, the bus internal data using asymmetric encryption mechanism, consumers and providers can use the public key encryption and decryption of incoming information, and carries on the score keeping a log, in order to better management of network monitoring system.

- Agent: is the node on the network. It stores the description of the various services, can be used to find the location of the various services. Service bus for each consumer and provider provides an agent, then consumers will only for your particular agent for point-to-point communication. Provider is the same, so as to make the service bus provide other services to the outside world to realize the complete package.

- $\quad$ Service management center: consists of two parts, business repository and registry. Business library service from the perspective of business management. It is the interface of management, contracts, the SLA (Service - Level Agreement), dependency, etc., its purpose is to help identify, design and development Service. Registry management services from a technical perspective, but not all the details of the management contract, but management at runtime using service need all the technical details of the deployment information, can be used to each service call routing to provide corresponding service system. 


\section{Summary}

SOA is widely used in the enterprise integration, this paper will its application in the field of network monitoring management, the combination of SOA and network security system, so as to improve the network monitoring management fragmented situation. Reference SOA application in the enterprise integration, through this infrastructure to build a service bus and makes different platform development of monitoring software can realize the interaction, share data information, better realize the management of the network.

\section{References}

[1] Zhang Yi, Cai Wan dong and Wang Ming. "The network monitoring model based on P2P study” J. Computer engineering and design. 2008 (24).

[2]Josuttis,N,M“SOA in Practice The Art of Distributed Design” M. O’RellyMedia, Inc.2007

[3] Heng-Yunzhang and hai-tao he. "Safety research and design of SOA system” J. Journal of sichuan institute of technology (natural science edition), 2009. (01).

[4]Josuttis,N,M.SOA in Practice The Art of Distributed Design [M],O’Relly Media, Inc.2007

[5] Cai Ting-you, etc.” enterprise application integration based on SOA architecture (EA1) research “M. Microcomputer information. 2007. 